\title{
RESEARCH
}

\section{Hybrid Selection as a Method of Increasing Mapping Power for Radiation Hybrids}

\author{
Hywel Bowden Jones
}

\author{
Department of Genetics, Downing Street, Cambridge CB2 3EH, United Kingdom
}

\begin{abstract}
Radiation hybrids have become a widely used tool for physical mapping. A drawback of the technique is that large numbers of hybrids are required to construct robust, high-resolution maps. The information contained within a panel of radiation hybrids is limited by the frequency of retention of chromosomal fragments from the donor cell line. In almost all experiments to date, the retention frequency has been below the optimal level; therefore, many hybrids are needed to produce high-quality maps. Because of the labor-intensive nature of large-scale mapping projects, it is important to make panels as small as possible. One method that has been adopted is to produce initially a large number of hybrids that are all typed with a few loci. Those hybrids showing satisfactorily high retention are admitted to the final panel and the rest are discarded. In this way, a panel of radiation hybrids with higher than expected retention can be created. Methods for conducting such a selection regime are discussed. To investigate the potential advantages of selecting hybrids based on their retention frequency, simulations were run under a variety of conditions. As expected panels with high retention (40\%) provided better mapping resources than panels with lower $(20 \%)$ retention. Beginning with an initial panel of 200 hybrids, comparisons of a random sample of 100 hybrids and the set of those 100 hybrids showing the highest marker retention demonstrated that selection may not be always the best strategy despite the increase in mean retention it yields. The selection of hybrids containing large numbers of fragments leads to an overestimation of the frequency of radiation-induced breaks. When breaks occur with high frequency (for example, when high radiation doses are used), the selection of hybrids leads to a loss of linkage and hence an inability to order the markers. As such, the merits of screening hybrids depends on both the radiation dose and the desired map resolution.
\end{abstract}

Radiation hybrids have become an established tool for human genome mapping (Walter and Goodfellow 1993; Leach and O'Connell 1995). Their principle advantage lies in the fact that nonpolymorphic markers can be mapped and therefore it is possible to integrate expressed sequence tags (ESTs) and candidate genes into a framework of highly polymorphic microsatellite markers (Hudson et al. 1995; Gyapay et al. 1996). It has also proved a useful framework for building yeast artificial chromosome (YAC) contigs (Hudson et al. 1995; Kumlien et al. 1996). The construction of radiation hybrids is necessarily complex, and many different methodologies have been adopted (for review, see Walter and Goodfellow 1993; Leach and O'Connell 1995). As yet, no consensus exists as to the most effective experimental design. Worse still, experience has shown that all radiation hybrid panels are not equal-experiments carried out under identical conditions using exactly the same protocol pro-

E-MAIL hbj@mole.bio.cam.ac.uk; FAX 01223-333992. duce panels that retain markedly different quantities of DNA from the donor cell (P.N. Goodfellow, unpubl.). This retention frequency is critical to the success or failure of a radiation hybrid panel. For human-hamster radiation hybrids, average retention usually varies between $5 \%$ and $30 \%$ [but is occasionally higher, for example, see Cox (1990)]. With fragment retention in this range large numbers of hybrids are required for the construction of robust, high-resolution maps. Because of the time-consuming nature of growing up hundreds of hybrid cell lines, methods for testing and improving the final set of hybrids have been proposed. One method is the "pooling" of hybrids, that is mixing the DNA from several different hybrid cell lines (Lunetta et al. 1995). This strategy aims to increase the mapping power by increasing the marker retention frequency. For example, pooling two haploid hybrids each with an average marker retention of $20 \%$ will give a single diploid hybrid line with a retention frequency of $36 \%$. Thus, it is hoped that fewer hybrids will be needed to gain the re- 


\section{JONES}

quired map resolution. This will mean both less work in growing up the panel and a reduction in the final number of hybrids that must be scored for the markers (therefore, reducing the number of PCRs). By repeatedly pooling the DNA, the resulting lines will have high ploidy and specific methods for analyzing this type of data have been developed (Lange et al. 1995; Lunetta et al. 1995).

An alternative method for improving the mapping power of a radiation hybrid panel is to impose a selection regime whereby only hybrids containing a certain quantity of human DNA are admitted to the final panel. Because the irradiation and fusion steps are relatively straightforward, a large number of hybrids (several hundred) are made initially. These are then all screened for the quantity of human DNA they carry. Those retaining little or no human DNA are discarded. This method of selecting hybrids with high retention has been used in a number of studies (e.g., James et al. 1994; Kumlien et al. 1996). The quantity of human DNA in a hybrid may be assessed using Alu-PCR or fluorescent in situ hybridization (FISH) (Warrington et al. 1991; Sinke et al. 1992; Francke et al. 1994; Kumlien et al. 1996). However, these processes can be extremely time consuming for large numbers of hybrids and it is difficult to set a quantitative cutoff point for the acceptance or rejection of a hybrid. If retention is very low, these methods represent an effective strategy for excluding hybrids that contain no human DNA.

A simpler method for screening a panel for retention is to type a small number of markers across all the hybrid cell lines and select those hybrids that are positive for a large proportion of the loci. The choice of markers depends on whether a single chromosomal region or the whole genome is to be mapped. If only a small region is of interest, for example, if a detailed map is needed in a positional cloning experiment, the markers used for screening should lie in close proximity to this area allowing hybrids not retaining human DNA from this region to be excluded. If the panel is to be used for many different purposes, the genome wide retention frequency will be of interest. Markers used to screen the hybrids should be unlinked (preferably from different chromosomes) to provide independent estimates of retention. Ideally at least one marker from each chromosome would be typed on the entire panel but this may be impractical if there are large numbers of hybrids. Factors such as the reported increase in retention near the centromere (Benham et al. 1989; Goodfellow et al. 1990; Lawrence et al. 1991; Ceccherini et al. 1992) should be taken into consideration when choosing markers for the purpose of screening.

A trade-off exists between the investment of time in screening the panel and the certainty that those hybrids selected genuinely do contain a larger than average proportion of the human genome. Treating the loci as independent and assuming that all loci have the same probability of retention $p$, the number of positive scores, $n$, from $N$ loci typed has a binomial distribution given by

$$
P(n)=\frac{N !}{(N-n) ! n !} p^{n}(1-p)^{N-n}
$$

for $0 \leqslant n \leqslant N$. For radiation hybrids made with a diploid donor cell line, $p$ will represent the probability of either copy of the marker being retained.

Given $n^{*}$ positive results from $N$ markers typed, equation 1 allows the calculation of confidence limits (say 95\%) for the true retention frequency of the hybrid. For each possible value of $n^{\star}$, Table 1 gives the confidence intervals such that the probability the true retention frequency is contained in the interval is $95 \%$ (left panel). The right panel gives the minimum value of retention such that the probability the true retention is greater than this value is $95 \%$. In practice, retention is usually in the region of $10 \%$ to $30 \%$ and therefore the requirement will be that a hybrid has retention greater than some minimum acceptable value.

In screening the hybrids for high marker retention there is a correlation between the number of markers typed and the certainty that a particular hybrid has satisfactorily high retention. Table 1 shows clearly that screening with a greater number of loci provides a more precise estimate of the true retention frequency of the hybrids as the size of the confidence intervals are greatly reduced. The optimal retention frequency is a function of both the distribution of the size of fragments and density of markers to be mapped. Intuitively it might be expected that a retention frequency of $50 \%$ would be optimal as hybrids retaining all or none of the loci are equally uninformative for mapping (Barrett 1992; Lange and Boehnke 1992; Lunetta and Boehnke 1994). If the fragment size and the retention frequency are not independent, then it may be better to sacrifice retention to produce hybrids with frag- 


\begin{tabular}{|c|c|c|c|c|c|c|}
\hline \multirow{3}{*}{$\begin{array}{l}\text { Number of loci } \\
\text { present in the } \\
\text { hybrid }\left(n^{*}\right)\end{array}$} & \multicolumn{6}{|c|}{ Number of loci typed $(N)$} \\
\hline & \multicolumn{3}{|c|}{$\begin{array}{l}\text { Two-tailed } 95 \% \text { confidence intervals for } \\
\text { genome-wide retention frequency }{ }^{\mathbf{a}}\end{array}$} & \multicolumn{3}{|c|}{$\begin{array}{l}\text { One-tailed } 95 \% \text { confidence interval for } \\
\text { genome-wide retention frequency }\end{array}$} \\
\hline & 5 & 10 & 20 & 5 & 10 & 20 \\
\hline 0 & $(0.00,0.52)$ & $(0.00,0.31)$ & $(0.00,0.17)$ & 0.00 & 0.00 & 0.00 \\
\hline 5 & $(0.48,1.00)$ & $(0.19,0.81)$ & $(0.09,0.49)$ & 0.54 & 0.22 & 0.10 \\
\hline 10 & - & $(0.69,1.00)$ & $(0.27,0.73)$ & - & 0.74 & 0.30 \\
\hline 15 & - & - & $(0.51,0.91)$ & - & - & 0.54 \\
\hline 20 & - & - & $(0.83,1.00)$ & - & - & 0.86 \\
\hline
\end{tabular}

ments of the appropriate size for the map resolution required. In the simulations that follow it will be assumed that retention is independent of the size of the fragments and therefore, it would be expected that a panel with an average retention frequency of $50 \%$ would be optimal. To test the effectiveness of such a selection scheme, a set of computer simulations were run under a variety of conditions to assess the mapping power (defined by a number of different criteria described in the Methods below).

\section{RESULTS}

As an initial test of the hypothesis that mapping power is maximized when the mean retention is close to $50 \%$, panels of hybrids were simulated where the probability of a fragment being retained was either $20 \%$ or $40 \%$. Both high and low radiation doses and equal and unequal distributions of markers were investigated. The results of 2000 replicates are summarized in Table 2 (see Methods). In all conditions and for all of the four

Table 2. High- and Low-retention Radiation Hybrid Panels

\begin{tabular}{|c|c|c|c|c|c|c|c|c|}
\hline \multirow[b]{3}{*}{$\begin{array}{l}\text { Average number of } \\
\text { orders within lod } 3 \text { of the } \\
\text { most likely order }\end{array}$} & \multicolumn{4}{|c|}{ Low dose } & \multicolumn{4}{|c|}{ High dose } \\
\hline & \multicolumn{2}{|c|}{$\begin{array}{l}\text { Equally } \\
\text { spaced loci }\end{array}$} & \multicolumn{2}{|c|}{$\begin{array}{l}\text { Unequally } \\
\text { spaced loci }\end{array}$} & \multicolumn{2}{|c|}{$\begin{array}{l}\text { Equally } \\
\text { spaced loci }\end{array}$} & \multicolumn{2}{|c|}{$\begin{array}{l}\text { Unequally } \\
\text { spaced loci }\end{array}$} \\
\hline & 1.04 & 1.00 & 1.44 & 1.13 & 1.08 & 1.01 & 2.48 & 1.94 \\
\hline $\begin{array}{l}\text { Percent of panels with }>1 \\
\text { order within lod } 3 \text { of the } \\
\text { most likely order }\end{array}$ & 3.8 & 0.5 & 35.5 & 13.4 & 6.7 & 0.7 & 72.9 & 60.3 \\
\hline $\begin{array}{l}\text { Average log-likelihood } \\
\text { difference between the } \\
\text { best and second best } \\
\text { orders }\end{array}$ & 2.04 & 2.37 & 1.75 & 2.03 & 2.19 & 2.53 & 1.68 & 1.93 \\
\hline $\begin{array}{l}\text { Percent most likely order } \\
\text { correct }\end{array}$ & 100 & 100 & 97.7 & 99.9 & 99.8 & 100 & 97.7 & 99.0 \\
\hline Observed retention (\%) & 20.0 & 40.1 & 20.0 & 40.0 & 20.0 & 40.0 & 20.0 & 40.0 \\
\hline
\end{tabular}


indicators of mapping power, the panels with $40 \%$ retention perform better than those with $20 \%$ retention (the sole exception being the percentage correct in the low dosage panels with equally spaced markers where both the $20 \%$ and $40 \%$ retention simulations place the true order first in all cases). It is also notable that the lowdose hybrids perform better than the high-dose hybrids in all cases. Finally, the equally spaced markers can be more reliably mapped for all four criterion than can the unequally spaced markers. In summary, for the simulations described here, low-dose, high-retention hybrids performed far better than high dosage hybrids with low marker retention. Furthermore, equally spaced markers can be mapped with more confidence than markers that are unevenly distributed across the chromosome.

As expected, hybrids with retention in the region of $40 \%$ proved a more effective tool for mapping than did hybrids with lower retention $(\sim 20 \%)$. Given that most hybrid panels produced to date have mean retention of $-20 \%$, further simulations were run to investigate the merits of selecting a subset of 100 hybrids that had higher than average retention. Tables 3 and 4 show the outcome (for low and high doses, respectively) for the full panel of 200 hybrids (full), a random subset of 100 hybrids (random), and a set containing the 100 hybrids with the highest retention (high).

For the low-dose simulations, the full panel of 200 hybrids performs at least as well and usually much better than either of the subsets, the single exception being in the average log-likelihood difference between the first and second orders where the high retention subset performs better than either the full panel or a random subset of that panel. For unevenly spaced markers, the full set gave slightly better results than the high retention subset and both were superior to random (see Table 3 ). These simulations provide evidence that the selection of hybrids for higher than average retention increase both the probability of obtaining the true order and the strength of support for this order when compared to a randomly selected subset of the same size. However, typing the full set provides greater mapping power than either of the subsets.

In the high-dose case, the expected number of breaks per chromosome is three times higher than in the previous simulations. Table 4 summarizes the results for 2000 replicates. When the markers are equally spaced across the chromosome, the full panel performs better than either of the subsets as before (although the high retention panel actually recovers the true order slightly more frequently than the full panel of 200 hybrids). However, the random subset now has fewer orders within a lod score of 3 (lod3) of the best as well as fewer panels with more than one order to lod 3 compared to the high retention subset. The subsets show very similar loglikelihood differences between the top two orders and the panel selected for high retention gives

Table 3. Low-dose Radiation Hybrid Panels

\begin{tabular}{|c|c|c|c|c|c|c|}
\hline \multirow[b]{2}{*}{ Low dose } & \multicolumn{3}{|c|}{ Equally spaced loci } & \multicolumn{3}{|c|}{ Unequally spaced loci } \\
\hline & all & random & high & all & random & high \\
\hline $\begin{array}{l}\text { Average number of orders } \\
\text { within lod } 3 \text { of the most likely order }\end{array}$ & 1.04 & 1.89 & 1.11 & 1.44 & 4.20 & 1.69 \\
\hline $\begin{array}{l}\text { Percent of panels with }>1 \text { order } \\
\text { within lod } 3 \text { of the most likely order }\end{array}$ & 3.8 & 56.8 & 9.8 & 35.5 & 90.0 & 47.8 \\
\hline $\begin{array}{l}\text { Average log-likelihood } \\
\text { difference between the best } \\
\text { and second best orders }\end{array}$ & 2.04 & 1.74 & 2.21 & 1.75 & 1.15 & 1.75 \\
\hline $\begin{array}{l}\text { Percent most likely order } \\
\text { correct }\end{array}$ & 100 & 96.7 & 100 & 97.7 & 79.9 & 97.1 \\
\hline Observed retention (\%) & 20.0 & 20.1 & 40.1 & 20.0 & 19.9 & 40.0 \\
\hline
\end{tabular}




\begin{tabular}{|c|c|c|c|c|c|c|}
\hline \multirow[b]{2}{*}{ High dose } & \multicolumn{3}{|c|}{ Equally spaced loci } & \multicolumn{3}{|c|}{ Unequally spaced loci } \\
\hline & all & random & high & all & random & high \\
\hline $\begin{array}{l}\text { Average number of orders within lod } 3 \text { of } \\
\text { the most likely order }\end{array}$ & 1.08 & 3.61 & 5.68 & 2.48 & 6.06 & 6.14 \\
\hline $\begin{array}{l}\text { Percent of panels }>1 \text { order within lod } 3 \text { of } \\
\text { the most likely order }\end{array}$ & 6.7 & 78.7 & 96.1 & 72.9 & 98.8 & 100 \\
\hline $\begin{array}{l}\text { Average log-likelihood difference between } \\
\text { the best and second best orders }\end{array}$ & 2.19 & 1.77 & 1.78 & 1.68 & 0.87 & 0.10 \\
\hline Percent most likely order correct & 99.8 & 96.6 & 99.9 & 97.7 & 84.7 & 65.4 \\
\hline Observed retention (\%) & 20.0 & 20.0 & 39.5 & 20.0 & 19.9 & 39.8 \\
\hline
\end{tabular}

the correct order as the most likely with greater frequency.

When trying to map unevenly distributed markers, the case is even more striking. Once again the full panel of 200 hybrids is better than either subset for all four indicators. However, the random subset of 100 hybrids performs better than the 100 hybrids selected for high retention. Particularly noticeable is the difference in the frequency with which the most likely order is correct. For the random subset this is $84.7 \%$, whereas for the selected subset (high), the proportion has dropped to $65.4 \%$. This is in marked contrast to the low-dose case where the selected hybrids proved to be better for all the indicators than the random subset.

Finally, to ensure that selecting those hybrids with the highest retention was not significantly different from the set of hybrids with retention closest to $50 \%$, a further 2000 radiation hybrid panels were simulated with equally spaced loci. Table 5 summarizes the results for the full panel of 200 hybrids (full), a random subset of 100 hybrids (random), the set of 100 hybrids with highest retention (high), and the set of 100 hybrids with retention closest to $50 \%$ (best). The comparisons between full, random, and high are as described previously (Tables 3 and 4). The subset of hybrids with retention closest to $50 \%$ (best) gives very similar results to the subset of hybrids with the highest retention (high). The latter perform slightly better for all the criteria at high dose and at least as well for all criteria except the average log-likelihood difference between the first two orders in the low-dose simulations. Overall there was no strong evidence to suggest selecting those hybrids with the highest locus retention is a worse strategy than choosing those hybrids with retention closest to $50 \%$ in the cases examined here.

Table 5 also summarizes the maximum likelihood estimates for the interlocus breakage frequency, $\theta$. A striking result is the increase in the estimation of $\theta$ in the selected subsets. The effect is particularly noticeable in the high-dose simulations where the probability of at least one break occurring between consecutive loci is in excess of $60 \%$ for the selected panels. This represents a significant overestimation of the probability of breakage (the true frequencies are $18 \%$ and $45 \%$ for low and high dose, respectively).

In summary, the high retention subset performed better than the random subset at the lower dose. However, this was reversed at the higher radiation dose. Sets of markers that are equally spaced are more easily mapped than markers where the interlocus distance varies along the chromosome, irrespective of the retention frequency or the size of the panel. Furthermore, recovery of the true map order was greater when the frequency of breakage was low (on average one per chromosome) than when it was high (three breaks per chromosome on average). For the distributions of markers considered here, selecting hybrids proved an effective strategy when breaks were infrequent but appeared to have a deleterious effect when the frequency of breakage was high. Thus mapping power appears 
Table 5. Selected Subsets of Hybrids with Retention Closest to 50\%

\begin{tabular}{|c|c|c|c|c|c|c|c|c|}
\hline & \multicolumn{4}{|c|}{ Low dose } & \multicolumn{4}{|c|}{ High dose } \\
\hline & full & random & high & best & full & random & high & best \\
\hline \multicolumn{9}{|l|}{ Average number of orders within } \\
\hline \multicolumn{9}{|l|}{ Percent of panels $>1$ order within } \\
\hline lod3 of the most likely order & 4.4 & 56.3 & 9.2 & 14.6 & 6.9 & 79.0 & 94.5 & 98.4 \\
\hline \multicolumn{8}{|l|}{ Average log-likelihood difference } & \\
\hline best orders & 2.02 & 1.76 & 2.19 & 2.24 & 2.16 & 1.78 & 1.78 & 1.41 \\
\hline Percent most likely order correct & 99.8 & 96.0 & 100 & 99.9 & 99.8 & 96.8 & 99.4 & 99.5 \\
\hline Observed retention (\%) & 20.0 & 20.0 & 39.9 & 27.9 & 20.0 & 20.0 & 39.5 & 35.5 \\
\hline Estimate of $\theta$ & 0.19 & 0.19 & 0.25 & 0.30 & 0.46 & 0.47 & 0.60 & 0.64 \\
\hline \multicolumn{6}{|l|}{ Average pairwise lod score for } & & & 32 \\
\hline $\begin{array}{l}\text { A summary of the results of simulations } \\
\text { subset of } 100 \text { hybrids (random), a subs } \\
\text { closest to } 50 \% \text { (best). Also shown are th th } \\
\text { scores for adjacent loci (s.E.M. }<0.06 \text { ir } \\
\text { respectively }(n=2000) \text {. }\end{array}$ & ases). & true $v$ & of $\theta$ ar & 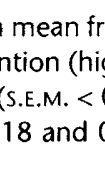 & for & $w$ and & dose & $\begin{array}{l}\text { random } \\
\text { etention } \\
\text { wise lod } \\
\text { ulations }\end{array}$ \\
\hline
\end{tabular}

to be a function of radiation dose (and hence fragment size), retention frequency, the number of hybrids, and the distribution of the loci to be mapped.

\section{DISCUSSION}

Contrary to expectations, the selection of 100 hybrids that have the highest retention does not necessarily provide a better tool for mapping than a randomly chosen subset of the same size. One possible explanation is that, because the hybrids were selected for maximum retention, a large number of them may have $>50 \%$ retention. In the extreme, a hybrid containing the entire human chromosome contains no information for mapping. If large numbers of hybrids were positive for all loci, then mapping would be difficult as there would not be enough breaks to clearly order the markers. This hypothesis is not supported by the fact that at low breakage frequencies, the selected panel proved more effective than the random subset. The expectation would be that when breaks occur very infrequently, a large number of hybrids would contain the entire chromosome and hence would be positive for all markers. At higher doses, the chromosome would be more fragmented and fewer hybrids should contain complete human chro- mosomes. Thus, it would be expected that the selection regime would be more effective at high radiation doses than at low ones, the opposite of what was observed. In addition, simulations showed that subsets of 100 hybrids with retention closest to $50 \%$ were no more effective than the high retention subsets.

Selecting hybrids with high retention implies selection of hybrids with either particularly large or particularly many fragments. In the high-dose case it will probably be the latter. This leads to an increase in the mean number of breaks per hybrid and hence to an inflation of the estimates of breakage frequency and interlocus distances. In the extreme, the increase in the estimate of the breakage frequencies leads to a loss of linkage between the markers; therefore, ordering them becomes difficult. This is borne out by average pairwise lod scores for neighboring loci (Jones 1996) shown in Table 5. As expected, the increased frequency of breakage in the high-dose simulations leads to lower pairwise lod scores in all cases. At the low dose, the evidence for linkage is stronger in the subset selected for high retention than in the random subset. This is reversed in the highdose simulations. The hybrids selected to have retention as close to $50 \%$ as possible have the lowest pairwise lod scores and this is reflected in their relatively poor mapping power. 


\section{SELECIED SUBSEIS OF RADIATION HYBRIDS}

In a similar manner, pooling high-dose radiation hybrids may also result in a loss of linkage. If two hybrids containing a number of small fragments are combined, the mean number of breaks per hybrid will be inflated in the same way as it is for the selection regime described above. However, a recent simulation study by Lunetta and co-workers (1995) found some benefit to pooling hybrids when retention was very low $(8 \%)$ and the average interlocus distance was $10-20 \mathrm{cR}$. More work is needed to confirm whether these results hold for a wider range of parameter values.

Of critical importance is resolution of map required (and hence the number of markers to be scored). If markers are to be placed at $10 \mathrm{Mb}$ intervals along the genome and the mean fragment size at a given radiation dose is $10 \mathrm{Mb}$, on average there will be only one locus per fragment. In this way, most of the fragments will be uninformative (the pairwise lod scores for neighboring loci will be very low) and mapping will prove extremely difficult. However, if markers are placed at $1 \mathrm{Mb}$ intervals across the genome, the fragments will on average contain 10 loci and it will be possible to determine linkage between the markers. Thus, it is vital that the dose at which a panel is created reflects the desired resolution of the map that is to be constructed.

Although the results presented here are by no means a comprehensive examination of the merits of hybrid selection, they highlight cases where selecting radiation hybrids on the basis of their retention frequency may not be an advantageous strategy. Methods to improve the average retention in a panel, either through pooling or a selection regime, must be treated with caution. Whether such techniques will increase the probability of recovering the true locus order or increase the support for this order will depend on both the radiation dose and the distribution of the markers. Any method of selection will bias the estimates of the parameters representing the breakage and fragment retention probabilities. Such unwanted biases may have a deleterious effect on the measurement of interlocus distances and these estimates should be treated with caution.

In most cases, selection may be an effective policy as it can greatly reduce the number of hybrids that must be scored. This is particularly true if only the order of the markers is of interest and not the distances. When retention is very low and many hybrids contain little or no human DNA, selection to exclude those hybrids will be a reasonable strategy for creating a radiation hybrid panel of a manageable size. However, it will always be better to type twice the number of hybrids if the resources are available.

\section{METHODS}

For simplicity, monochromosomal hybrids were simulated under the assumption that breakage occurs as a Poisson process. Retention was assumed to be equal for all fragments irrespective of their size or the chromosomal location from which they came. Furthermore it was assumed that the probability of a fragment being retained in a given hybrid was unaffected by the number of fragments the hybrid had already retained. The simulated chromosome was 50 units long and two different distributions of six markers were considered. In the first (the equally spaced scheme), the markers where placed every 10 units along the length of the chromosome. In the second (the unequally spaced scheme) the markers were separated by distances $20,4,2,4$ and 20 units running from telomere to telomere. In addition, two radiation doses were considered. The first has a mean number of breaks per chromosome of 1 (Poisson process with intensity, $\lambda=1 / 50$ ) and the second had a mean number of breaks per chromosome of $3(\lambda=3 / 50)$. These will be termed the low- and highdose treatments, respectively. Fragment retention frequency was set at $20 \%$ unless otherwise stated.

For each run, 200 hybrids were simulated under the assumptions given above (all). A random subset of 100 hybrids were then taken (random). Finally those 100 hybrids with the highest retention were selected (high). For clarity, selection takes place under the assumption of perfect knowledge about the number of loci retained in each hybrid (but not about the number of fragments). These markers are not independent estimates of retention as they are all located on the same chromosome and therefore, are linked to some degree. In practice, the small number of markers typed across the entire panel would be used to infer which hybrids had the highest retention. As the number of loci used to screen the panel decreases, the selected subset regresses to being a random subset of 100 hybrids. Analysis was carried out using multipoint maximum likelihood methods under the assumption that fragments are retained with equal probability (Boehnke et al. 1992, 1991; Chakravarti and Reefer 1992; M. Boehnke, E. Hauser, K. Lange, J. Uro, and J. Vander Stoep, unpubl.).

The aim of the simulations was to assess the mapping power of a selected panel compared to both a random subset of the same size and to the full panel that contains twice as many hybrids. Here the term mapping power is used to encompass both the ability to recover the true order from the data and the confidence with which this order is favored over other possible locus orders. Comparisons between orders is provided by the logarithm (base 10) of the ratio of likelihoods of the two orders [this is an extension of the lod score method (Morton 1955)]. A lod3 implies that one order is a thousand times more likely than the other (see Ott 1992 and references therein).

Four criteria were used to assess the mapping power of the radiation hybrid panels:

(1) the average number orders within lod 3 of the most likely order; 
(2) the frequency of panels having more than one order within lod 3 of the most likely order;

(3) the average log-10 likelihood difference between the first and second most likely orders (assuming there is another order within lod3 of the most likely); and

(4) the frequency with which the most likely order is correct.

These criteria are not necessarily independent but in combination provide a measure of the power of a panel of hybrids to recover the true map order. It is important that they not only suggest the correct ordering of the markers, but also that they indicate that it is much more likely than any other possible order.

Each set of simulations consisted of 2000 independent hybrid panels. This was enough to provide good estimates of the criterion defined above. In all conditions, the standard error of the mean (S.E.M.) was $<0.1$ for both the average number of orders within lod 3 of the best and the average log-10 likelihood difference between the top two orders (criteria 1 and 3 above). For the frequency of panels having more than one order within lod 3 of the most likely and the frequency of the best order being correct (criteria 2 and 4), the S.E.M. was $<1 \%$ in all sets of simulations. Finally, the estimation of the retention frequency had S.E.M. $<1 \%$.

\section{ACKNOWLEDGMENTS}

Thanks to Peter Goodfellow and Kathryn Lunetta for comments on earlier versions of this manuscript. This work was supported by the Medical Research Council, UK.

\section{REFERENCES}

Barrett, J.R. 1992. Genetic mapping based on radiation hybrid data. Genomics 13: 95-103.

Benham, F., K. Hart, J. Crolla, M. Bobrow, M. Francavilla, and P.N. Goodfellow. 1989. A method for generating hybrids containing nonselected fragments of human chromosomes. Genomics 4: 509-517.

Boehnke, M., K. Lange, and D.R. Cox. 1991. Statistical methods for multipoint radiation hybrid mapping. Am. J. Hum. Genet. 49: 1174-1188.

Ceccherini, I., G. Romeo, S. Lawrence, M.H. Breuning, P.C. Harris, H. Himmelbauer, A.M. Frischauf, G.R. Sutherland, G.G. Germino, S.T. Reeders, and N.E. Morton. 1992. Construction of a map of chromosome 16 by using radiation hybrids. Proc. Natl. Acad. Sci. 89: 104-108.

Chakravarti, A. and J.E. Reefer. 1992. A theory for radiation hybrid (Goss-Harris) mapping: Applications to proximal 21q markers. Cytogenet. Cell Genet. 59: 99-101.

Cox, D.R., M. Burmeister, E.R. Price, S. Kim, and R.M. Myers. 1990. Radiation hybrid mapping: A somatic cell genetic method for consturctin high-resolution maps of mammalian chromosomes. Science 250: 245-250.

Francke, U., E. Chang, K. Comeau, E.M. Geigle, J. Ciacalone, X. Li, J. Luna, A. Moon, S. Welch, and P. Wilgenbus. 1994. A radiation hybrid map of human chromosome 18. Cytogenet. Cell Genet. 66: 196-213.

Goodfellow, P.J., S. Povey, H.A. Nevanlinna, and P.N. Goodfellow. 1990. Generation of a panel of somatic cell hybrids containing unselected fragments of human chormosome 10 by X-ray irradation and cell fusion: application to isolating the MEN2A region in hybrid cells. Somat. Cell Mol. Genet. 16: 163-171.

Gyapay, G., K. Schmitt, C. Fizames, H.B. Jones, N. Vega-Czarny, D. Spillet, D. Muselet, J.-F. Prud'Homme, C. Dib, C. Auffray, J. Morrissette, J. Weissenbach, and P.N. Goodfellow. 1996. A radiation hybrid map of the human genome. Hum. Mol. Genet. 5: 339-346.

Hudson, T., L. Stein, S. Gerety, J. Ma, A. Castle, J. Silva, D. Slonim, R. Baptista, L. Kruglyak, S. Xu, X. Hu, A. Colbert, C. Rosenberg, M. Reeve-Daly, S. Rozen, L. Hui, X. Wu, C. Vestergaard, K. Eilson, J. Bae, S. Maitra, S, Ganiatsas, C. Evans, M. DeAngelis, K. Ingalls, R. Nahf, L. Horton, M. Oskin, A. Collymore, W. Ye, V. Kouyoumjian, I. Zernsteva, J. Tarn, R. Devine, D. Courtney, M. Renaud, H. Nguyen, T. O'Connor, C. Fizames, S. Faure, G. Gyapay, C. Dib, J. Morissette, J. Orlin, B. Birren, N. Goodman, L. Weissenbach, T. Hawkins, S. Foote, D. Page, and E. Lander. 1995. An STS based map of the human genome. Science 270: 1945-1955.

James, M.R., C.W. Richard, J.J. Schott, C. Yoursy, K. Clark, J. Bell, J.D. Terwilliger, J. Hazan, C. Dubay, A. Vignal, M. Agrapart, T. Imai, Y. Nakamura, M. Polymeropoulos, J. Weissenbach, D.R. Cox, and G.M. Lathrop. 1994. A radiation hybrid map of 506 STS markers spanning human chromosome 11. Nature Genet. 8: $70-76$.

Jones, H.B. 1996. Pairwise analysis of radiation hybrid mapping data. Ann. Hum. Genet. 60: 351-357.

Kumlien, J., A. Grigoriev, H. Roest Crollius, M. Ross, P.N. Goodfellow, and H. Lehrach. 1996. A radiation hybrid map spanning the entire human $X$ chromosome integrating YACs, genes and STS markers. Mamm. Genome (in press).

Lange, K. and M. Boehnke. 1992. Bayesian methids and optimal experimental design for gene mapping by radiation hybrids. Ann. Hum. Genet. 56: 119-144.

Lange, K., M. Boehnke, D.R. Cox, and K.L. Lunetta. 1995. Statistical analysis for polyploid radiation hybrid mapping. Genome Res. 5: 136-150.

Lawrence, S., N.E. Morton, and D.R. Cox. 1991. Radiation hybrid mapping. Proc. Natl. Acad. Sci. 88: $7477-7480$. 


\section{SELECTED SUBSETS OF RADIATION HYBRIDS}

Leach, R.J. and P. O'Connell. 1995. Mapping of mammalian genomes with radiation (Goss and Harris) hybrids. Adv. Genet. 33: 63-99.

Lunetta, K.L. and M. Boehnke. 1994. Multipoint radiation hybrid mapping: Comparison of methods, sample size requirements, and optimal study characteristics. Genomics 21: 92-103.

Lunetta, K.L., M. Boehnke, K. Lange, and D.R. Cox. 1995. Experimental design and error detection for polyploid radiation hybrid mapping. Genome Res. 5: 151-163.

Morton, N.E. 1955. Sequential tests for the detection of linkage. Am. J. Hum. Genet. 7: 277-318.

Ott, J. 1992. Analysis of human genetic linkage. John Hopkins University Press, Baltimore, MD.

Sinke, R.J., F.F. Suijkerbuijk, J. Herbergs, H. Janssen, J.J. Cassiman, and A. Geurts van Kessel. 1992. Generation of a panel of somatic cell hybrids containing fragments of human chromosome $12 \mathrm{p}$ by X-ray irradiation and cell fusion. Genomics 12: 206-213.

Walter, M.A. and P.N. Goodfellow. 1993. Radiation hybrids: Irradiation and fusion gene transfer. Trends Genet. 9: 352-356.

Warrington, J.A., L.V. Hall, L.M. Hinton, J.N. Miller, J.J. Wasmuth, and M. Lovett. 1991. Radiation hybrid map of 13 loci on the long arm of chromosome 5 (published erratum appears in Genomics 1992 14: 832). Genomics 11: 701-708.

Received March 18, 1996; accepted in revised form June 11, 1996. 


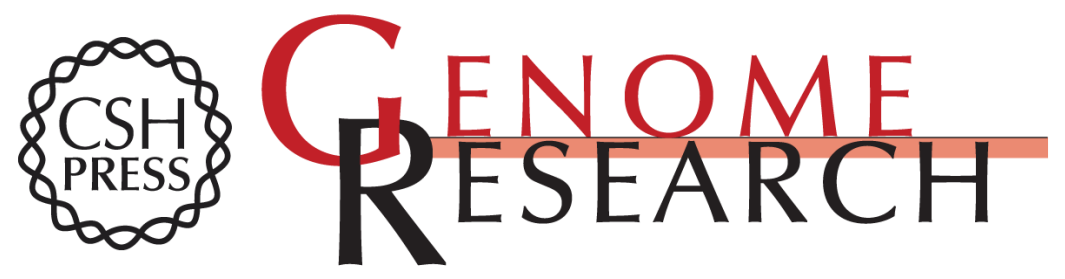

\section{Hybrid selection as a method of increasing mapping power for radiation hybrids.}

H B Jones

Genome Res. 1996 6: 761-769

Access the most recent version at doi:10.1101/gr.6.8.761

References This article cites 22 articles, 6 of which can be accessed free at:

http://genome.cshlp.org/content/6/8/761.full.html\#ref-list-1

\section{License}

Email Alerting Receive free email alerts when new articles cite this article - sign up in the box at the Service top right corner of the article or click here.

\section{Affordable, Accurate Sequencing.}

To subscribe to Genome Research go to: https://genome.cshlp.org/subscriptions 